\title{
Usefulness of Elevated Troponin to Predict Severity in Patients with COVID-19
}

\author{
KAZI ALAM NOWAZ, AFM SHAMSUL HAQUE, SYEDA ALEYA SULTANA, MOHAMMAD ABDUL MALEK, \\ WALIUR RAHMAN, MOSTAFA KAMAL, MD. SANAUL HAQUE, JINAT FARJANA \\ MD. NOORNABI KHONDAKER \\ Department of Cardiology, Combined Military Hospital (CMH), Dhaka \\ Address of Correspondence : Major (Dr.) Kazi Alam Nowaz, Department of Cardiology, CMH, Dhaka. \\ Email: kanjewel@gmail.com
}

\begin{abstract}
Background: Coronavirus disease 2019 (COVID-19) has produced a significant health burden worldwide. Recent studies had shown that cardiac injury in COVID-19 is not uncommon and may leads to death. The aim of this study was to assess cardiac troponin in patients with COVID-19 and to evaluate the incidence of myocardial injury.

Methods: A cross-sectional study was designed among 100 hospitalized patients with moderate to severe COVID-19 in Combined Military Hospital (CMH), Dhaka from January to April, 2021, after considering inclusion and exclusion criteria, study population was divided into two groups on the basis of normal and elevated troponin level.

Results: Participants had mean age of $67.33 \pm 7.63$ years with $76 \%$ men. $42 \%$ population had elevation in troponin levels. Risk factors like age, Diabetes (DM), C-reactive protein (CRP), Lactate dehydrogenase (LDH), D- Dimer, Ferrtin level were more in elevated troponin group and statistically significant. Troponin levels significantly increased with increasing severity of COVID-19 staging (Kendall's tau-b $=0.479, \mathrm{p}=0.004$ ) with reflected moderate positive correlation. Multivariate analysis was performed for association of COVID19 severity with elevated troponin separately after adjusting for other known confounding factors. Independent association between COVID-19 severity and troponin remained significant after that along with other acute phase reactants and inflammatory markers.

Conclusion: COVID-19 can significantly leads to myocardial injury. This data suggested elevated troponin level is one of the determinants of clinical outcome in patients with COVID-19.
\end{abstract}

Keywords: COVID-19 severity; Myocardial injury; Troponin I

University Heart Journal 2022; 18(1): 29-35

\section{Introduction}

COVID-19 caused by severe acute respiratory syndrome coronavirus-2 (SARS-CoV2) remains a pandemic, with considerable mortality and morbidity exerting pressure on global health-care systems. ${ }^{1}$ Outbreaks lead to scarcity of hospital beds and shortage of medical equipment, while medical person including doctors themselves had been also become infected. Several countries have had or are experiencing third waves, and despite vaccination swiftly progress from mild constitutional symptoms to severe acute respiratory distress syndrome and multi-organ failure. ${ }^{4}$ Patients with COVID-19 experienced a wide range of disease severity. Prognostic tools that efficiently stratify individual's risk of experiencing adverse outcomes may facilitate patients and clinicians in the informed decision-making process. ${ }^{5}$ Despite being primarily a respiratory infection, COVID- 19 has important impacts on many vital organs, including the heart. ${ }^{6,7}$ Many studies had documented myocardial injury with COVID-19 reflected by elevated circulating cardiac troponin concentrations. ${ }^{8-12}$ However, increased troponin value frequently occur in patients with conditions other than acute coronary syndrome and the mechanisms are complex. For this reason, the American College of Cardiology recommended that troponin should be ordered for COVID-19 patients being considered on clinical backgrounds. ${ }^{5}$ Furthermore, it remained unclear that elevated troponin levels were due to ischemic myocardial injury via atherosclerotic plaque rupture or coronary thrombotic events (type 1 myocardial infarction) or indirect injury associated with and sepsis resulting in supply- demand mismatch (type 2 myocardial infarction), or a direct result of the inflammatory surge frequently observed in patients hospitalized with COVID-19 ${ }^{13}$. The rise in high sensitivity troponin I correlated with other inflammatory biomarkers (D-dimer, ferritin, interleukin- 
6, LDH), raising the possibility that this reflects cytokine storm or secondary hemophagocytic lymphohistiocytosis more than isolated myocardial injury. ${ }^{14}$ Elevated troponin value was common and predictive for subsequent death in hospitalized COVID 19 patients. ${ }^{5}$ Elevated troponin was significantly associated with fatal outcomes. ${ }^{15}$ Whatever the underlying mechanisms and pathophysiology of cardiac involvement in COVID-19, early assessment of cardiac biomarkers may be used to differentiate high-risk patients - who require intense monitoring and aggressive treatment, from low-risk participants. Thus, in times of limitation of resources and difficulties in priority setting low-cost and widely available cardiac biomarker may play a significant role. ${ }^{16}$ So, aim of this study was to find out correlation of troponin I and COVID-19 severity.

\section{Materials and Methods}

This cross-sectional analytical study was conducted in the Department of Medicine, CMH, Dhaka from January to April in 2021. We enrolled 100 adult (age $>18$ years) of hospitalized COVID-19 patients. We included patients of COVID-19 who admitted with of moderate to severe condition according to National guidelines. ${ }^{17}$ Patients age 18 years and older were initially stratified into two groups based on their cardiac troponin-I (elevated vs. normal). A normal cardiac troponin-I level was defined as $\leq 48 \mathrm{pg} / \mathrm{ml}$, which falls below the 99th percentile in the blood test based on our facility's laboratory data. An elevated high sensitivity troponin-I value was defined as $>48 \mathrm{pg} / \mathrm{ml}$. We excluded patients with ST-elevated myocardial infraction (STEMI), significant ECG changes suggestive of non ST- elevated myocardial infraction (NSTEMI), known structural heart disease and chronic kidney disease

\subsection{Statistical analyses}

Continuous variables were expressed as mean \pm SD and compared using Student's t test. Categorical variables were presented as counts and percentages and compared with x2-test when appropriate. Correlation between COVID19 and troponin I had been done and plotted by box plot separately. First, univariate analysis were included in multivariate logistic regression. Then, multivariate analysis based on a bidirectional elimination were performed for association of severity of COVID-19 in order to take into account the higher correlation that may exist between some variables particularly those of comorbidities and of laboratory markers. Results were reported as odds ratio (ORs) and 95\% confidence intervals (CIs). A two-sided p value $<0.05$ was considered statistically significant. All analyses were performed using SPSS (Statistical programed for social science) statistical software version 16.0.

\section{Results}

A total of 100 patients were enrolled for the study: there were 42 patients in group I (High sensitivity Troponin I d" $48 \mathrm{pg} / \mathrm{ml}$ ) and 58 patients in group II (High sensitivity Troponin I $>48 \mathrm{pg} / \mathrm{ml}$ ). This study participants had predominance of age group of 60 to 70 years with mean age of $67.33 \pm 7.63$ years with $76 \%$ men. A total of 58 patients $(58 \%)$ had normal troponin and $42(42 \%)$ had elevation in troponin levels. There were significant differences in baseline characteristics between two troponin groups (Table I).

Table I

Baseline Characteristics of Patients with COVID-19 Based on the Cardiac Troponin-I level

\begin{tabular}{|c|c|c|c|}
\hline & $\begin{array}{c}\text { Group I } \\
\text { Troponin I } 48 \& \\
\text { below }(n=58)\end{array}$ & $\begin{array}{c}\text { Group II } \\
\text { Troponin I } \\
\text { above } 48(n=42)\end{array}$ & $\begin{array}{c}\text { P Value \& } \\
\text { Levels of sig. }\end{array}$ \\
\hline \multicolumn{4}{|l|}{ Gender } \\
\hline Male & $76.2 \%$ & $75.9 \%$ & $0.581^{\mathrm{NS} *}$ \\
\hline Female & $23.8 \%$ & $24.1 \%$ & \\
\hline HTN & $54.8 \%$ & $57.6 \%$ & $0.471^{\mathrm{NS}} *$ \\
\hline DM & $33.3 \%$ & $51.7 \%$ & $0.049^{S^{*}}$ \\
\hline Age & $66.62 \pm 8.82$ & $68.00 \pm 5.59$ & $0.02^{\mathrm{S} * *}$ \\
\hline S. Creatinine & $1.07 \pm .24$ & $1.14 \pm .25$ & $.873 \mathrm{NS} * *$ \\
\hline LDH & $316 \pm 62.31$ & $431 \pm 121.35$ & $0.001^{\mathrm{S}^{* *}}$ \\
\hline CRP & $11.3 \pm 5.56$ & $13.9 \pm 3.66$ & $<0.001^{\mathrm{S}^{* *}}$ \\
\hline BNP & $692 \pm 105.71$ & $732 \pm 132.69$ & $0.410 \mathrm{NS} * *$ \\
\hline D-Dimer & $0.6 \pm 0.30$ & $0.9 \pm 0.21$ & $<0.001^{\mathrm{S}^{* *}}$ \\
\hline HRCT of Chest & $34.76 \pm 6.21$ & $54.68 \pm 12.61$ & $0.001^{\mathrm{S}^{* *}}$ \\
\hline S. Ferritin & $448 \pm 103.67$ & $476 \pm 136.75$ & $0.001^{\mathrm{S}^{* *}}$ \\
\hline Troponin I & $16.92 \pm 16.92$ & $1056 \pm 735.66$ & $<0.001^{\mathrm{S}^{* *}}$ \\
\hline
\end{tabular}

NS=NOT SIGNIFICANT, S= SIGNIFICANT * CHI SQUARE TEST DONE, ** STUDENT'S T TEST DONE HTN: Hypertension; DM: Diabetes mellitus; CRP:C reactive protein; $\mathrm{LDH}$ : Lactate dehydrogenase 
Older Patients had more troponin level than younger patients $(66.62 \pm 8.82$ vs. $68.00 \pm 5.59, \mathrm{p}<0.05$ for normal vs. elevated troponin). Study showed that male patients were predominant in both groups $(76.2 \%$ vs. $23.8 \%$ in group I and $75.9 \%$ vs. $24.1 \%$ in group II). Statistically significant difference was not seen in terms of sex among the groups $(\mathrm{p}=0.581)$. Most hospitalized patients had hypertension (HTN) but statistically significant difference was not seen between two groups. Diabetic (DM) patients were more prevalent in elevated troponin I group $(\mathrm{p}<$ 0.05 ) and statistically significant. There was a consistent and significant increase in the degree of elevated acute phase reactants and inflammatory markers in elevated troponin group. Compared with patients with normal troponin, patients with elevated troponin had a higher level of CRP (11.3 \pm 5.56 vs. $13.9 \pm 3.66 ; p<0.05)$, D-dimer ( $0.6 \pm 0.3$ vs. $0.9 \pm 0.21 ; p<0.05)$, and serum ferritin level ( $448 \pm 103.67$ vs. $476 \pm 136.75 ; p<0.05)$. In addition, $L D H$ also elevated in group II ( $316 \pm 62.31$ vs. $431 \pm 121.35$; p $<0.05$ ). High Resolution CT scan of chest showed more involvement of lungs in elevated troponin group (34.76 \pm 6.21 vs. $54.68 \pm 12.61 ; \mathrm{p}<0.05)$ which was statistically significant. Brain natriuretic peptide (BNP) was elevated in group II but statistically was not significant $(692 \pm 105.71$ vs. $732 \pm 132.69 ; \mathrm{p}>0.05$ ). Of these patients, a significantly greater percentage of patients had elevated troponin $(58 \%$ had normal and $42 \%$ had elevated troponin; $p<0.05$ among groups. Figure I showed the escalation of troponin I was positively correlate with $\mathrm{LDH}$, a marker of coagulopathy.

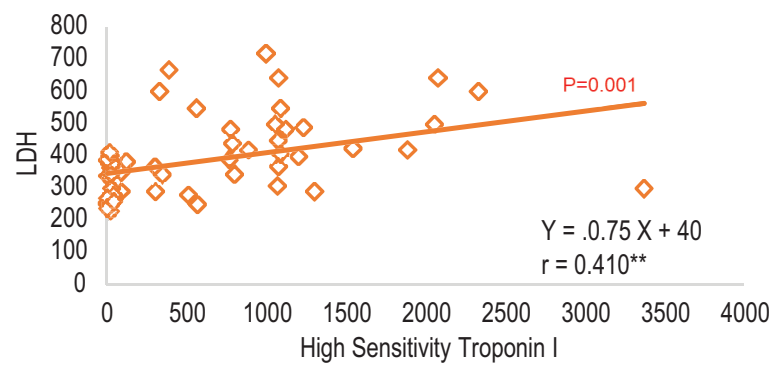

Fig.-1: Correlation between $\mathrm{LDH}$ and troponin I level among the study population

Troponin I levels significantly increased with increasing severity of COVID-19 as determined by clinical status staging (Kendall's tau-b correlation coefficient ô (100) $=0.479, p=0.004$ two tailed) with reflected moderate strength of positive correlation (Fig 2).

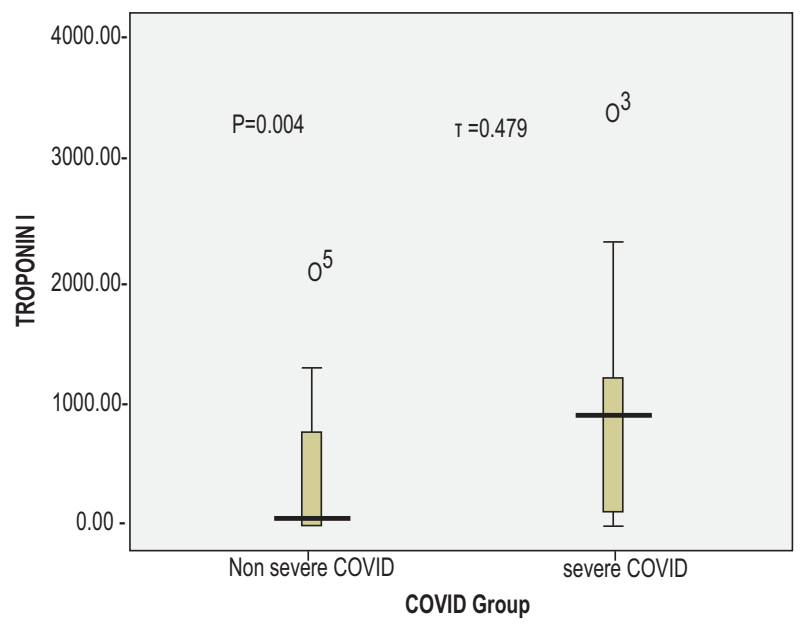

Fig.-2: Correlation between COVID Clinical staging and High Sensitivity Troponin I represented by Simple boxplot

Receiver operating characteristic (ROC) curve of troponin I $(\mathrm{AUC}=0.860, \mathrm{P}<0.001)$ was shown in (Fig III), the best cut-off point for severe illness was $667 \mathrm{pg} / \mathrm{mL}$ with a specificity of $76.7 \%$ and a sensitivity of $21.4 \%$. According to ROC curves of troponin for diagnosis of COVID-19 severity area under curve (AUC) 0.860 which reflected that larger result of test variable indicate stronger evidence for a positive actual state.

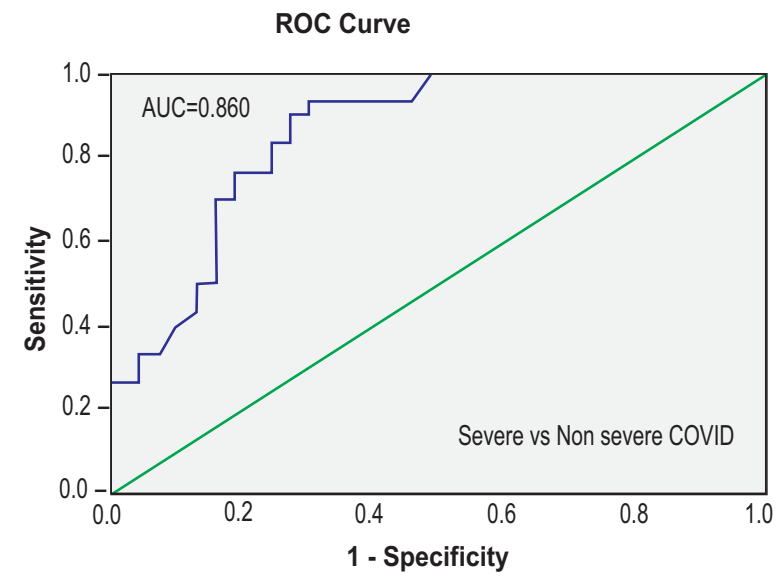

Fig.-3: Receiver operating characteristic (ROC) curves of troponin for the diagnosis of COVID severity

Then at first, univariate regression analysis was performed which showed association of COVID-19 severity with DM, LDH, CRP, D-Dimer, serum Ferritin and BNP level along with troponin (OR 2.084, 95\% CI 1.18-0.989, $\mathrm{p}=$ $0.003)$. Then a multivariate regression analysis was also performed to see the association of COVID severity to other confounding factors (Table 2). Independent association was persisted with Troponin I with COVID severity (OR 2.044, 95\% CI 1.004-1.000, $\mathrm{p}=0.032$ ) along with LDH, CRP, D-Dimer and serum Ferritin level. 
Table II

Binary logistic regression analysis of different factors for clinical COVID-19 severity

\begin{tabular}{|c|c|c|c|c|}
\hline & $\begin{array}{c}\text { Univariate model } \\
\text { OR }(95 \% \mathrm{CI})\end{array}$ & $\bar{p}$ & $\begin{array}{c}\text { Multivariate model } \\
\text { OR }(95 \% \mathrm{CI})\end{array}$ & $\bar{p}$ \\
\hline Sex & $0.374(2.049-0.255)$ & $0.541^{\mathrm{NS}}$ & & \\
\hline Age & $0.329(1.076-0.961)$ & $0.566^{\mathrm{NS}}$ & & \\
\hline HTN & $0.923(3.599-0.645)$ & $0.337^{\mathrm{NS}}$ & & \\
\hline DM & $0.903(1.172-0.634)$ & $0.004^{\mathrm{S}}$ & $0.903(17.21-0.634)$ & $0.926^{\mathrm{NS}}$ \\
\hline S. Creatinine & $0.83(1.706-0.880)$ & $0.973^{\mathrm{S}}$ & & \\
\hline LDH & $2.4(0.658-0.142)$ & $0.003^{\mathrm{S}}$ & $2.3(1.031-1.001)$ & $0.02^{\mathrm{S}}$ \\
\hline Troponin I & $2.084(1.18-0.989)$ & $0.003^{\mathrm{S}}$ & $2.044(1.004-1.000)$ & $0.032^{\mathrm{S}}$ \\
\hline S. Ferritin & $2.94(0.784-0.157)$ & $0.004^{\mathrm{S}}$ & $2.750(1.018-0.993)$ & $0.03^{\mathrm{S}}$ \\
\hline CRP & $1.484(0.646-0.321)$ & $0.001^{\mathrm{S}}$ & $1.035(0.70-1.43)$ & $0.04^{\mathrm{S}}$ \\
\hline D-Dimer & $2.5(0.658-0.342)$ & $0.001^{\mathrm{S}}$ & $2.19(1.11-1.28)$ & $0.01^{\mathrm{S}}$ \\
\hline $\mathrm{BNP}$ & $1.21(1.335-1.104)$ & $0.03^{\mathrm{S}}$ & $0.624(1.011-0.995)$ & $0.430^{\mathrm{NS}}$ \\
\hline
\end{tabular}

NS=Not Significant, S= Significant OD: odd ratio; CI: confidence interval; HTN: Hypertension; DM: Diabetes mellitus; CRP:C reactive protein; LDH: Lactate dehydrogenase

\section{Discussion}

Important findings of this study were i) COVID-19 severity was associated with increased troponin level. Association persists after adjusting for other factors known to affect these variables. ii) There was an incremental increase in acute phase reactants, inflammatory markers and markers of coagulopathy that correlated with elevated troponin.

There were many studies which showed body of data regarding the prevalence and impact of myocardial injury in patients hospitalized with COVID-19. In recent studies of small number of patient of COVID-19 estimated that myocardial injury occurred in approximately $30 \%$ of patients, ranging from $20 \%$ to $50 \% .{ }^{18-23}$ The presence of elevated troponin, or its dynamic increase during hospitalization, increased up to 5 times the risk of requiring ventilation, also increased in arrhythmias such as ventricular tachycardia/ventricular fibrillation, and 5 times the risk for mortality. ${ }^{18}$ In this study, approximately $42 \%$ of patients who had evidence of myocardial injury as reflected by elevation in troponin of patients with moderate to severe COVID. Compelling evidence has shown that cardiac troponin elevations are strongly associated with disease severity and hard outcomes in patients with COVID-19, irrespective of the underlying mechanism of cardiovascular involvement. ${ }^{16}$ Acute myocardial injury is typically seen in advanced stages of disease, the possible mechanisms of myocardial injury include: i) hyper inflammation and cytokine storm mediated through pathologic $\mathrm{T}$ cells and monocytes which leads to myocarditis. ${ }^{24}$

iii) respiratory failure and hypoxemia which resulting in damage to cardiacmyocytes, ${ }^{25}$ iv) down regulation of ACE2 expression and subsequent protective signaling pathways in cardiac myocytes, v) hypercoagulability by which development of coronary microvascular thrombosis, ${ }^{26} \mathrm{vi}$ ) diffuse endothelial injury and 'endotheliitis' in several organs, including the heart as a direct consequence of SARS-CoV-2 viral involvement and/or resulting from host inflammatory response, ${ }^{27}$ and, vii) inflammation and/or stress causing coronary plaque rupture or supply-demand mismatch leading to myocardial ischemia/infarction (MI). In the study by Oudit et al., ${ }^{28}$ the SARS-CoV viral RNA was detected in 35\% (7/20) of autopsied human heart samples obtained from patients who died during the SARS outbreak. Due to presence of ACE2 receptors on cardiac myocytes, direct infiltration of cardiac myocardium by SARS-CoV-2 virus is also a possibility. Other suggested mechanisms of COVID-19 related heart injury include cytokine storm, mediated by increased proinflammatory cytokine production by innate immunity after COVID-19 infection, and hypoxia induced excessive intracellular calcium which leads to myocyte apoptosis $^{29,30}$. COVID-19 seems to affect the myocardium and cause myocarditis. Interstitial mononuclear inflammatory infiltrates in myocardium had been documented in death cases of COVID-19. ${ }^{31}$ Furthermore, 
cases of myocarditis with reduced systolic function have been reported after COVID- 19 infection. ${ }^{32}$ In the setting of COVID-19, myocardial injury, defined by an elevated troponin level, occurs especially due to non-ischaemic myocardial processes, including severe respiratory infection with hypoxia, sepsis, systemic inflammation, pulmonary thrombosis and embolism, cardiac adrenergic hyper stimulation during cytokine storm syndrome, and myocarditis. ${ }^{33}$

The incidence of myocardial injury increased with age and was disproportionately associated with male sex ${ }^{13}$. In this study, also elevated troponin I was found in elderly people and became statistically significant and predominant was male but which was not statistically significant. As per earlier studies, ${ }^{34}$ in this study, diabetic patients had significant elevated troponin I whether hypertension was not statistically significant. Several studies have shown that cardiovascular involvement and elevated cardiac troponin levels were present in patients with severe COVID-19 compared to those with non- severe COVID-19. ${ }^{33,35,36}$ Furthermore, the same studies revealed that myocardial damage was associated with higher levels of inflammatory biomarkers; more severe lung involvement; increased need for non-invasive and invasive ventilation; and increased rates of ARDS and bleeding disorders. In this study, also showed that there was a correlation with elevated troponin with others levels of inflammatory biomarkers like CRP, serum ferritin level, lung involvement by HRCT of chest and also D-Dimer and LDH level. This elevated troponin reflects myocardial injury resulting from the associated cytokine storm manifested by elevated levels of CRP, ferritin, lactate dehydrogenase (LDH), and D-dimer or myocardial dysfunction from the direct effect of severe acute respiratory syndrome coronavirus on the heart. ${ }^{14}$

In a recent systematic review of four studies including 374 patients, cardiac troponin levels were significantly elevated in those with severe COVID-19 infection in comparison with those with non-severe disease. ${ }^{37}$ In this study, multivariate regression analysis was performed to see the impact of elevated troponin on COVID 19 after analysis of other known confounding factors responsible for COVID severity. Troponin remained significant with CRP, LDH, D-Dimer, serum ferritin level and thus conforming earlier similar findings. ${ }^{34,38,39}$ It is therefore reasonable, in accordance with data of this study, to perform cardiac troponin measurement for SARS- CoV-2 infection. This could identify high-risk patients who could be the target of advanced therapies and timely treatment.
This study has many limitations. First, the associations shown in our study while significant were only modest. This was due to small sample size, single-center experience and cross-sectional purposive nature of the study. Secondly, although multivariate analysis was performed in this study, there may still be other confounding factors that can only be addressed in the prospective studies.

\section{Conclusion}

This single center small study showed that more than forty percent of the patients studied with COVID-19 had elevated values of troponin along with more severe symptoms and clinical course of the disease. This result strongly recommended that measuring high sensitivity cardiac troponin in patients diagnosed with COVID-19 for early diagnosis of complications and therefore timely treatment. As the COVID-19 pandemic continues to hit developing countries like us with thousands of hospitalizations and deaths, the availability of low cost tools with an established predictive value may wisely support frontline physicians in predicting early severity.

\section{Statement of ethics}

This study was approved by ethical committee of $\mathrm{CMH}$, Dhaka after submission of protocol.

\section{Source of Funding}

None

\section{Author contributions}

KAN conceptualized the idea, drafted protocol, KAN and MNK collected data, did literature search and drafted the manuscript. SA did the guidance. SYA, AM, MK, SA and JF were involved in patient management. All the authors read and approved final manuscript for submission.

\section{Statement of authorship}

All the authors whose names are listed above had taken responsibility for all aspects of the reliability and freedom from bias of the data presented and their discussed interpretation.

\section{Acknowledgements}

We acknowledged all patients who were involved in this study.

\section{Conflicts of interest:}

Nothing to declare.

\section{References}

1. Wynants L, Van Calster B, Collins GS, Riley RD, Heinze G, Schuit E, et al. Prediction models for diagnosis and prognosis of COVID19: Systematic review and critical appraisal. BMJ. 2020;369:115. 
2. Looi MK. COVID-19: Is a second wave hitting Europe? BMJ. 2020;371:4113.

3. Woolf SH, Chapman DA, Lee JH. COVID-19 as the Leading Cause of Death in the United States. JAMA - J Am Med Assoc. 2021;325(2):123-24.

4. Giustino G, Pinney SP, Lala A, Reddy VY, Johnston-Cox HA, Mechanick JI, Halperin JL, Fuster V. Coronavirus and Cardiovascular Disease, Myocardial Injury, and Arrhythmia: JACC Focus Seminar. J Am Coll Cardiol. 2020;76(17):2011-23

5. Zhao BC, Liu WF, Lei SH, Zhou BW, Yang X, Huang TY, et al. Prevalence and prognostic value of elevated troponins in patients hospitalised for coronavirus disease 2019: a systematic review and meta-analysis. J Intensive Care. 2020;8(1):1-15.

6. Guzik TJ, Mohiddin SA, Dimarco A, Patel V, Savvatis K, MarelliBerg FM, et al. COVID-19 and the cardiovascular system: Implications for risk assessment, diagnosis, and treatment options. Cardiovasc Res. 2020;116(10):1666- 87.

7. Liu PP, Blet A, Smyth D, Li H. The Science Underlying COVID19: Implications for the Cardiovascular System. Circulation. 2020;2019:68-78.

8. Bavishi C, Bonow RO, Trivedi V, Abbott JD, Messerli FH, Bhatt DL. Special Article - Acute myocardial injury in patients hospitalized with COVID-19 infection: A review. Prog Cardiovasc Dis. 2020;63:682-89.

9. Li JW, Han TW, Woodward M, Anderson CS, Zhou H, Chen YD, Neal B.The impact of 2019 novel coronavirus on heart injury: A Systematic review and Meta-analysis. Prog Cardiovasc Dis. 2020;63(4):518-24.

10. Li X, Guan B, Su T, Liu W, Chen M, Bin Waleed K, et al. Impact of cardiovascular disease and cardiac injury on in-hospital mortality in patients with COVID-19: A systematic review and meta-analysis. Heart. 2020;106(15):1142-47.

11. Parohan M, Yaghoubi S, Seraji A. Cardiac injury is associated with severe outcome and death in patients with Coronavirus disease 2019 (COVID-19) infection: A systematic review and meta-analysis of observational studies. Eur Hear Journal Acute Cardiovasc Care. 2020;9(6):665-77.

12. Santoso A, Pranata R, Wibowo A, Al-Farabi MJ, Huang I, Antariksa B. Cardiac injury is associated with mortality and critically ill pneumonia in COVID-19: A meta- analysis. Am J Emerg Med. 2020;19:1-6.

13. Majure DT, Gruberg L, Saba SG. Usefulness of Elevated Troponin to Predict Death in Patients With COVID-19 and Myocardial Injury. 2020;(138):100- 106.

14. Clerkin KJ, Fried JA, Raikhelkar J, Sayer G, Griffin JM, Masoumi A, et al. COVID-19 and Cardiovascular Disease. Circulation. 2020;2019:1648-55.

15. AL Abbasi B, Torres P, Ramos-Tuarez F, Dewaswala N, Abdallah A, Chen K, et al. Cardiac Troponin-I and COVID-19: A Prognostic Tool for In-Hospital Mortality. Cardiol Res. 2020;11(6):398-404.

16. Cannata F, Bombace S, Stefanini GG. Cardiac biomarkers in patients with COVID-19: pragmatic tools in hard times. Rev Esp Cardiol. 2021;1-3.
17. National Guidelines on Clinical Management of COVID-19. 2020;(November):20-29.

18. Guo T, Fan Y, Chen M, Wu X, Zhang L, He T, et al. Cardiovascular Implications of Fatal Outcomes of Patients with Coronavirus Disease 2019 (COVID-19). JAMA Cardiol. 2020;5(7):811-18.

19. Shi S, Qin M, Shen B, Cai Y, Liu T, Yang F, et al. Association of Cardiac Injury with Mortality in Hospitalized Patients with COVID-19 in Wuhan, China. JAMA Cardiol. 2020;5(7):802-10.

20. Lala A, Johnson KW, Januzzi JL, Russak AJ, Paranjpe I, Richter F, et al. Prevalence and Impact of Myocardial Injury in Patients Hospitalized With COVID-19 Infection. J Am Coll Cardiol. 2020;76(5):533-46.

21. Huang C, Wang Y, Li X, Ren L, Zhao J, Hu Y, et al. Clinical features of patients infected with 2019 novel coronavirus in Wuhan, China. Lancet. 2020;395(10223):497-506.

22. Wang D, Hu B, Hu C, Zhu F, Liu X, Zhang J, et al. Clinical Characteristics of 138 Hospitalized Patients with 2019 Novel Coronavirus-Infected Pneumonia in Wuhan, China. JAMA - J Am Med Assoc. 2020;323(11):1061-69.

23. Perrone MA, Spolaore F, Ammirabile M, Romeo F, Caciagli P, Ceriotti F, et al. The assessment of high sensitivity cardiac troponin in patients with COVID-19: A multicenter study. IJC Hear Vasc. 2021;32:100715

24. Zhou Y, Fu B, Zheng X, Wang D, Zhao C, Qi Y, et al. Pathogenic T-cells and inflammatory monocytes incite inflammatory storms in severe COVID-19 patients. Natl Sci Rev. 2020;7(6):998- 1002.

25. Kubasiak LA, Hernandez OM, Bishopric NH, Webster KA. Hypoxia and acidosis activate cardiac myocyte death through the Bcl-2 family protein BNIP3. Proc Natl Acad Sci U S A. 2002;99(20):12825-830.

26. Han H, Yang L, Liu R, Liu F, Liu F, Wu KL, et al. Prominent changes in blood coagulation of patients with SARS-CoV-2 infection. Clin Chem Lab Med. 2020;58(7):1116-20.

27. Varga Z, Flammer AJ, Steiger P, Haberecker M, Andermatt R, Zinkernagel AS, et al. Endothelial cell infection and endotheliitis in COVID-19. Lancet. 2020;395(10234):1417-18.

28. Oudit GY, Kassiri Z, Jiang C, Liu PP, Poutanen SM, Penninger $\mathrm{JM}$, et al. SARS- coronavirus modulation of myocardial ACE2 expression and inflammation in patients with SARS. Eur J Clin Invest. 2009;39(7):618-25.

29. Zhou F, Yu T, Du R, Fan G, Liu Y, Liu Z, et al. Clinical course and risk factors for mortality of adult inpatients with COVID-19 in Wuhan, China: a retrospective cohort study. Lancet. 2020; 395(10229):1054-62.

30. Zheng YY, Ma YT, Zhang JY, Xie X. COVID-19 and the cardiovascular system. Nat Rev Cardiol. 2020;17(5):259-60.

31. Xu Z, Shi L, Wang Y, Zhang J, Huang L, Zhang C, et al. Pathological findings of COVID-19 associated with acute respiratory distress syndrome. Lancet Respir Med. 2020;8(4): $420-22$.

32. Madjid M, Safavi-Naeini P, Solomon SD, Vardeny O. Potential Effects of Coronaviruses on the Cardiovascular System: A Review. JAMA Cardiol. 2020;5(7):831-40. 
33. Imazio M, Klingel $\mathrm{K}$, Kindermann I, Brucato A, De Rosa FG, Adler Y, et al. COVID-19 pandemic and troponin: Indirect myocardial injury, myocardial inflammation or myocarditis? Heart. 2020;106(15):1127-31.

34. Chen C, Yan JT, Zhou N, Zhao JP, Wang DW. Analysis of myocardial injury in patients with COVID-19 and association between concomitant cardiovascular diseases and severity of COVID-19. Zhonghua Xin Xue Guan Bing Za Zhi.2020;48(7): $567-71$.

35. Boukhris M, Hillani A, Moroni F, Mansour S, Zhao X, Ybarra F, et al. Cardiovascular Implications of the COVID-19 Pandemic: A Global Perspective. Can J Cardiol. 2020;36:1068-80.
36. Long B, Brady WJ, Koyfman A, Gottlieb M. Cardiovascular complications in COVID-19. Am J Emerg Med.2020;38(7): 1504-07.

37. Lippi G, Lavie CJ, Sanchis-Gomar F. Cardiac troponin I in patients with coronavirus disease 2019 (COVID-19): Evidence from a meta-analysis. Prog Cardiovasc Dis. 2020;63:390-91.

38. Bonow RO, Fonarow GC, O'Gara PT, Yancy CW. Association of Coronavirus Disease 2019 (COVID- 19) With Myocardial Injury and Mortality. JAMA Cardiol. 2020;5:751-53.

39. Chen C, Chen C, Yan JT, Zhou N, Zhao JP WD. Analysis of myocardial injury in patients with COVID-19 and association between concomitant cardiovascular diseases and severity of COVID-19. Zhonghua Xin Xue Guan Bing Za Zhi. 2020;48(7):567-71. 